\title{
Effect of Encoder Eccentricity on Rolling Speed Control and Elimination Method
}

\author{
Ren-Tao ZHAO ${ }^{1, a}$, Xin-Long $\mathrm{SHI}^{1}$ and Zi WANG ${ }^{2}$ \\ ${ }^{1}$ School of electrical and control engineering, North China University of Technology, Beijing 100144, China \\ ${ }^{2}$ State Grid Beijing Maintenance Company, Beijing 100054, China
}

\begin{abstract}
Aiming to solve the problems of incremental photoelectric encoder eccentricity in the speed control system of rolling mill, this paper deduces the calculation model of actual speed, and gives a high precision approximation calculation method. Furthermore, the effectiveness and accuracy of the simplified model are verified by the simulation results. The online identification method of eccentricity and initial angle is also given in the paper. In addition, on the basis of continuous model, this article derives from the discrete model of traditional speed measurement scheme based on digital control system. This proposed algorithm has simplicity and excellent application value, and the effectiveness of the algorithm has been verified.
\end{abstract}

\section{Introduction}

Although the rapid development of the sensorless vector control system made a result that the application of speed feedback link in AC drive field is decreasing, roller speed regulation system which has wide-range and high-precision regulating speed still require to utilize incremental photoelectric encoder (referred to as optical encoder). Besides, DC speed regulating system still be widely used in speed closed loop system structure. According to the closed-loop control theory, the low frequency disturbance of feedback channel will have bad influence on the operation of the system, which may cause low-frequency oscillation of system. A simple eccentricity correction algorithm given in literature [1] can effectively overcome the low frequency disturbance caused by eccentricity of speed generator. But it is not suitable for digital system with incremental photoelectric encoder as speed feedback. Literature [2] uses SIMULINK to establish a simulation model of optical encoder, analyzes reasons about high-frequency noise of the velocity measurement of an encoder and their impact on system performance, and proposes a method to estimate the instantaneous speed of motor with speed observer. Although this method has a strong ability to suppress high-frequency noise, it still cannot eliminate the low-frequency signal generated by eccentricity. Literature [3] analyzes the expression forms of incremental optical encoder eccentricity, gives the realization method of eliminating eccentric effect by adding the diameter digital quantities. But the prerequisite of implementation of compensation is to add a set of photoelectric detection component in optical encoder. In order to eliminate the influence of eccentricity on velocity, we add two signals with 180 degrees phase difference in the external microprocessor together, which can play a very good

${ }^{a}$ Corresponding author: Ray_zhao@263.net 
compensation effect.

Based on the derivation of the velocity eccentricity model, this article proposes a simple velocity correction algorithm which can obtain ideal effect speed compensation without special modification of optical encoder.

\section{Establishment of eccentric model}

As shown in Figure 1, assuming that the center position of the motor shaft is located at $O$, and the optical encoder center is at O', photoelectric detection device of optical encoder is installed in the horizontal axis direction, the margin of the grating disk in optical encoder and the horizontal axis intersect at $\mathrm{P}$ point. Postulating that $\mathrm{OP}$ is equal to $\rho$, and $\theta_{0}$ is the angle between the photoelectric element that produces the $\mathrm{Z}$ pulse and the line OP, which will be a constant as soon as the optical encoder is installed $[4,5]$.

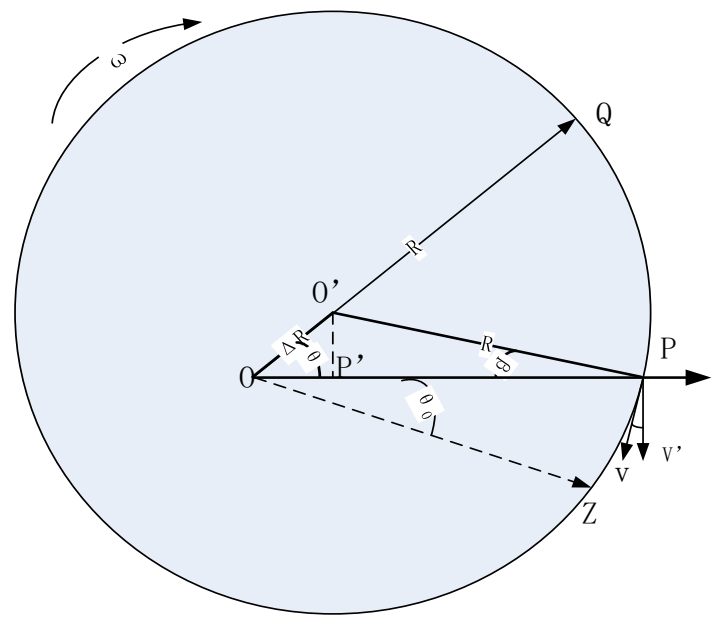

Figure 1. Diagram of eccentricity during the optical encoder installation process.

When the motor operates at angular speed $\omega$, the center point $\mathrm{O}^{\prime}$ of the optical encoder and the $\mathrm{Q}$ point on the edge of optical encoder rotate are around $O$ point at the same angular speed $[6,7]$. If the feature points' position on the optical encoder are shown in figure 1 at t time, the line OQ advances the horizontal axis by $\theta$ angle. According to the structure of the optical encoder, the frequency of pulse output from signal A or B is proportional to the line speed of P point (the tangential direction of eccentric circle) at any moment [8]. Accordingly, so long as the relation between OP and angle $\theta$ can be found out, it can be obtained that the relationship between optical encoder output frequency and angular velocity in the case of eccentric condition. The specific derivations will be given as follows.

This paper analyzes the situation that the optical encoder radius is $30 \mathrm{~mm}$, eccentric distance is $0.35 \mathrm{~mm}$. In order to derive mathematical model which is irrelevant to the radius of optical encoder, the radius $\mathrm{R}$ is normalized to 1 .

According to the sine theorem, we can know that

$$
\frac{R}{\sin \theta}=\frac{\Delta R}{\sin \beta} .
$$

Another form is that 


$$
\sin \beta=\frac{\Delta R \cdot \sin \theta}{R}
$$

This formula can be further derived:

$$
O P=O P^{\prime}+P^{\prime} P=\Delta R \cdot \cos \theta+R \cdot \cos \beta .
$$

According to the formula (2), we can find out that

$$
\cos \beta=R \sqrt{1-\left(\frac{\Delta R \cdot \sin \theta}{R}\right)^{2}}=\sqrt{R^{2}-\Delta R^{2} \sin ^{2} \theta} .
$$

Next, insert the equation (4) into equation (3), we can obtain equation (5) as follows after calculation.

$$
O P=\Delta R \cdot \cos \theta+\sqrt{R^{2}-\Delta R^{2} \sin ^{2} \theta}
$$

Combined with figure 1, we can get an equation about the relation between optical encoder output frequency and angular velocity $\omega$, as shown as formula (6).

$$
f=K \cdot \frac{V^{\prime}}{2 \pi R}=K \cdot \frac{V \cdot \cos \beta}{2 \pi R}=K \cdot \frac{O P \cdot \omega \cdot \cos \beta}{2 \pi R} .
$$

Among the formula (6), $\mathrm{K}$ is the number of pulses output from optical encoder for per revolution, namely the number of lines.

After that, insert the rotate speed formula $n=\frac{60 f}{K}$ into the formula (6), we can obtain the relation between rotate speed $n^{\prime}$ and OP under the eccentric condition.

$$
n^{\prime}=K \cdot \frac{60 \cdot O P \cdot \omega \cdot \cos \beta}{2 \pi R \cdot K}=9.55 \frac{O P}{R} \cdot \omega \cdot \cos \beta
$$

And then, take equation (5) into equation (7), we can finally obtains the function relationship between the $n^{\prime}$ with the angle $\theta$, as shown as equation (8).

$$
n^{\prime}=9.55 \frac{\left(\Delta R \cos \theta+\sqrt{R^{2} \Delta-R^{2} \sin ^{2} \theta}\right)}{R} \cdot \omega \cdot \frac{\sqrt{R^{2}-\Delta R^{2} \sin ^{2} \theta}}{R} .
$$

Evidently, the equation (8) can be simplified as equation (9), when the optical encoder is installed without deviating from the center $(\Delta R=0)$.

$$
n^{\prime}=\frac{60}{2 \pi} \cdot \omega=n
$$

In order to analyze the influence of eccentricity easily, we define the relative eccentricity as

$$
e=\frac{\Delta R}{R}
$$

From Figure 2 we can see that the frequency fluctuation degree caused by optical encoder 
eccentricity is $0 \sim \pm \frac{\Delta R}{R}= \pm 10 \%$, which means that the frequency fluctuation caused by eccentricity depends entirely on the relative eccentricity $e$.

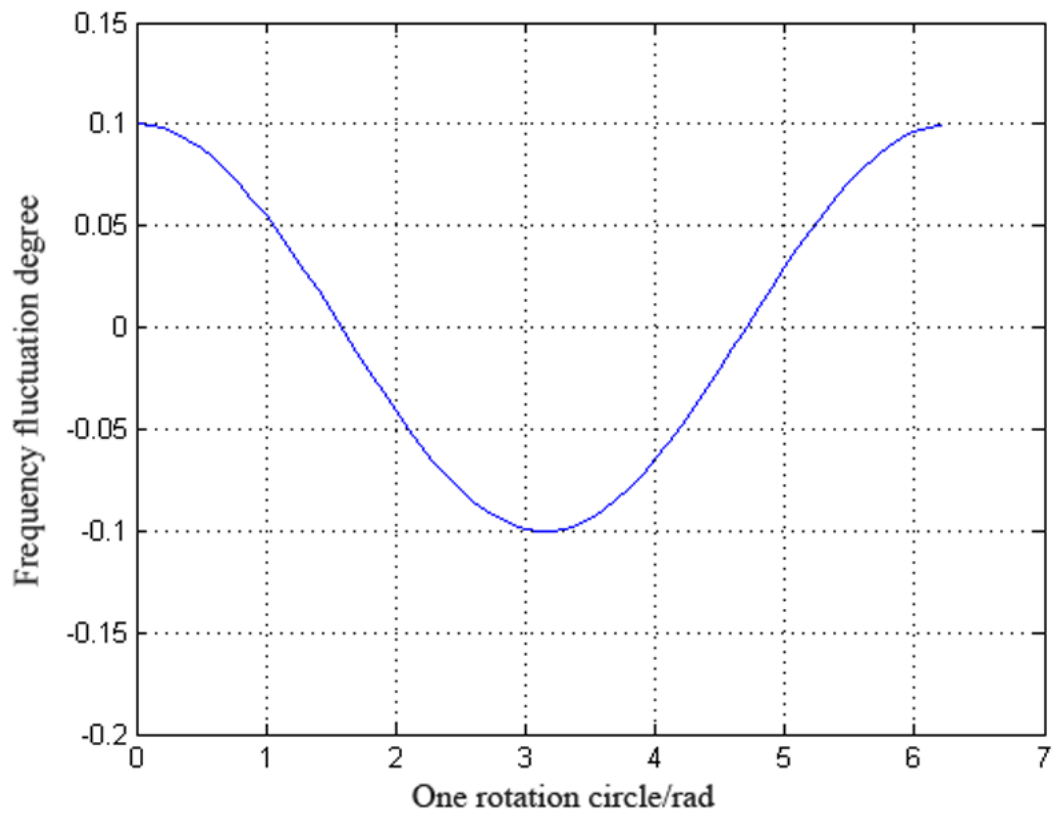

Figure 2. Normalized curve of optical encoder output frequency changing with angle $\theta$ for every rotation circle at constant speed ( $\mathrm{K}=1024, \omega=10.9, \Delta R=0.00035$ meters, $\mathrm{R}=0.03$ meters ) .

\section{Simplification of Eccentric Model}

Aiming to apply the above model to the actual speed regulation system, the equation (8) should be simplified firstly to cut down the amount of computation, in order to facilitate real-time velocity correction to eliminate the effect caused by eccentricity [4].

Assuming relative eccentricity $e$ is known, the relationship between the angle $\beta$ and $e$ can be calculated according to equation (2) and equation (10).

$$
\sin \beta=e \cdot \sin \theta .
$$

According to the Taylor series of $\sin \beta$ and $\cos \beta, \sin \beta$ can be approximately replaced by angle $\beta$ when $\beta$ is very small. Thus, the formula (2) can be adapted as

$$
\begin{aligned}
& \beta \approx e \cdot \sin \theta . \\
& \cos \beta=\sqrt{1-\sin ^{2} \beta} \approx \sqrt{1-\beta^{2}} \approx 1-\frac{1}{2} \beta^{2} .
\end{aligned}
$$


When the eccentricity reaches $0.125 \%$, it is less than $5 \mathrm{ppm}$ that the error caused by approximate calculation with (13), which can be ignored. And put formula (10) and (11) into the formula (6), we can further get formula (14) by ignoring $\frac{1}{2} \beta^{2}$ and $\frac{1}{4} \beta^{4}$.

$$
f \approx \frac{K}{2 \pi} \cdot\left(e \cdot \cos \theta+1-\beta^{2}\right) \cdot \omega
$$

The formula (7) is predigested as

$$
n \approx \frac{n^{\prime}}{\left(e \cdot \cos \theta+1-e^{2} \cdot \sin ^{2} \theta\right)} .
$$

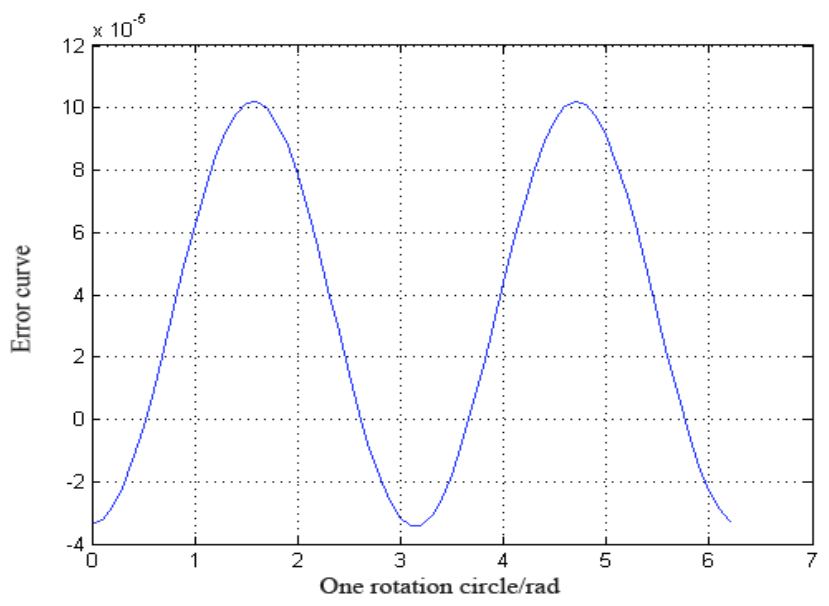

Figure 3. Error curve between the exact and approximate values of the rotate speed n' in a circle.

As can be seen from figure 3 , the results of preliminary simplification are quite satisfactory. The maximum absolute error is less than $1.02 * 10^{-4} \mathrm{rpm}$, which is equivalent to the $0.001 \%$ of current speed $104 \mathrm{rpm}(\omega=10.9)$, completely meet the high-precision velocity measurement.

Further simplification of the formula (15) leads to the simplest model as follows:

$$
n \approx \frac{n^{\prime}}{\left(e \cdot \cos \theta+1-0.25 \cdot e^{2}\right)} .
$$




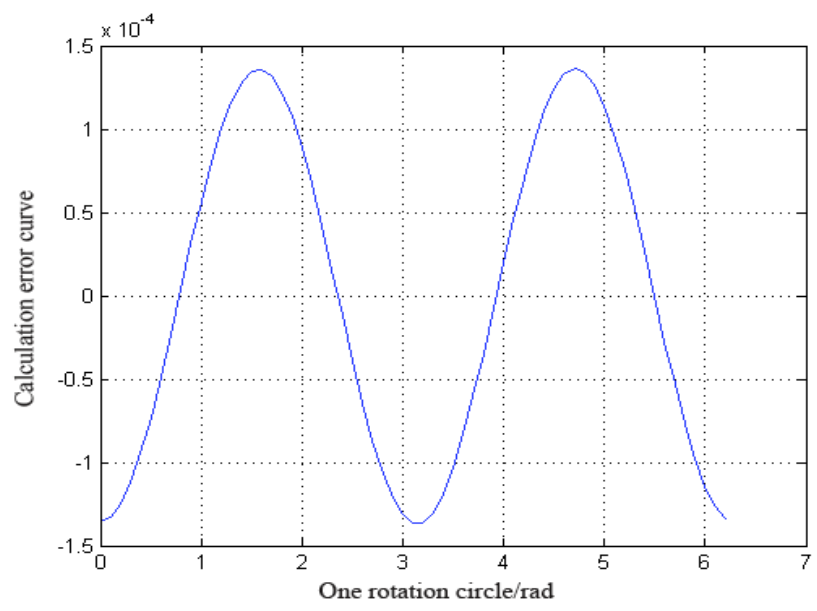

Figure 4. Calculation error curve of secondary simplification.

After observation of figure 4, we can find that the calculation error of the secondary simplified rotational speed model increases obviously. The maximum amplitude is close to $1.5^{*} 10^{-4} \mathrm{rpm}$, which is around $0.0014 \%$ of the current speed $104 \mathrm{rpm}$, and satisfy the requirements of higher precision velocity measurement.

\section{Online identification method of eccentricity}

Identification should be carried out in the case of no-load or constant load after the installation of optical encoder, for the purpose of ensuring the accuracy of identification.

(1) The output signal A of optical encoder should be connected to the pulse input port of the counter of microprocessor, and connects the signal $\mathrm{Z}$ to the input port of external interrupt.

(2) Motor operates at constant speed ( $\mathrm{n}=100 \sim 500 \mathrm{rpm})$.

(3) Start online identification program.

a) When the microprocessor detects the $\mathrm{Z}$ pulse for the first time, it starts the counting and frequency catching process automatically, and stops this process automatically at the time of detecting $\mathrm{Z}$ pulse secondly. In the meantime, record the maximum cycle $\mathrm{T}_{\max }\left(k_{1}\right)$ and minimum cycle $\mathrm{T}_{\min }\left(k_{2}\right)$ of signal A and the corresponding counter values $k_{1}$ and $k_{2}$. According to figure 1, P and Q position coincide at $k_{2}$ moment, $\mathrm{P}$ is precisely on the $\mathrm{OQ}$ extension line at $k_{1}$ moment.

b) Calculate the highest frequency $f_{\max }$ and the lowest frequency $f_{\min }$ separately on the basis of $\mathrm{T}_{\max }$ $\left(k_{1}\right)$ and $\mathrm{T}_{\min }\left(k_{2}\right)$.

$$
f_{\max }=\frac{1}{T_{\min }}, f_{\min }=\frac{1}{T_{\max }} .
$$

c) Convert $k_{1}$ and $k_{2}$ into angle, as follows.

$$
\theta_{1}=\frac{2 \pi \cdot k_{1}}{K}, \theta_{2}=\frac{2 \pi \cdot k_{2}}{K} .
$$

d) Calculate initial angle $\theta_{0}$ and relative eccentricity $e$.

$$
\theta_{0}=\left(\theta_{2}+\left|\pi-\theta_{1}\right|\right) / 2 \text {. }
$$


And $e$ can be obtained through formula (15).

$$
e=\left|\frac{f_{\max } / f_{\min }-1}{\cos \theta_{1}-\cos \theta_{2} \cdot f_{\max } / f_{\min }}\right| .
$$

Because the microprocessor chip is used in most of the speed regulating system [9], this paper needs to derive a recursive algorithm which can be directly applied to digital control system. The specific iterative algorithm is given as follows.

Assuming that the sampling period of velocity is fixed as $T_{s}$, the corresponding angular positions of the two adjacent sampling time are $\theta_{k}$ and $\theta_{k+1}$. Accordingly, the results of the sampling can be expressed with the following integral expression:

$$
n^{\prime \prime}=\frac{1}{T_{s}} \int_{\theta_{k}}^{\theta_{k+1}} n^{\prime} \cdot d \theta=\frac{n}{T_{s}} \int_{\theta_{k}}^{\theta_{k+1}}\left(e \cos \theta+1-\beta^{2}\right) \cdot d \theta .
$$

Put formula (12) into the above formula, we can get that

$$
n^{\prime \prime} \approx \frac{n}{T_{s}} \int_{\theta_{k}}^{\theta_{k+1}}\left(e \cdot \cos \theta+1-e^{2} \cdot \sin ^{2} \theta\right) \cdot d \theta=n \cdot F(\theta) .
$$
is

In this formula, $n$ is the result of speed calculation in the case of no eccentric, disturbance quantity

$$
F(\theta)=\frac{e}{T_{s}} \cdot\left(\sin \theta_{k+1}-\sin \theta_{k}\right)-\frac{e^{2}}{2 T_{s}}\left(\theta_{k+1}-\theta_{k}\right)+\frac{e^{2}}{4 T_{s}} \cdot\left(\sin 2 \theta_{k+1}-\sin 2 \theta_{k}\right) .
$$

Assuming that $M(k)$ is stand for the pulse count value at $\mathrm{k}$ moment and $M(k+l)$ is the pulse count value at $k+1$ moment in the digital control system. And both of them take $\mathrm{K}$ as modulus, which means that $0<M(k) \leq M(k+1)<K$. Then we can get equation (24) and equation (25). The actual rotational speed $n_{0}^{\prime \prime}(k)$ of any sampling period can be acquired by inserting equation (24) and (25) into formula (22).

$$
\begin{aligned}
& \theta_{k}=2 \pi \frac{M(k)}{K} . \\
& \theta_{k+1}=2 \pi \frac{M(k+1)}{K} . \\
& n_{0}^{\prime \prime}(k)=\frac{n^{\prime \prime}(k)}{F(k)} .
\end{aligned}
$$

The disturbance after discretization is

$$
\begin{aligned}
& F(k)=\frac{e}{T_{s}} \cdot\left(\sin \left(2 \pi \frac{M(k+1)}{K}\right)-\sin \left(2 \pi \frac{M(k)}{K}\right)\right)-\frac{\pi e^{2}}{T_{s} K}(M(k+1)-M(k)) \\
& +\frac{e^{2}}{4 T_{s}} \cdot\left(\sin \left(4 \pi \frac{M(k+1)}{K}\right)-\sin \left(4 \pi \frac{M(k)}{K}\right)\right)
\end{aligned} .
$$

Because sine function can be cured to the proceeding space in advance, the perturbation $F(k)$ that seems cumbersome in the preceding paragraphs can be completely converted to simple algebraic 
operations, which basically does not increase the computational burden to digital control systems.

\section{Simulation and Verification}

In the working process of rolling mill, low frequency disturbance of rotational speed caused by optical encoder eccentricity will have a great impact on product quality, such as steel plate creep deformation, uneven thickness. Meanwhile, the energy consumption of the rolling mill is increased obviously, and production decreased. Long time operation with fluctuation speed also shortens the life of the equipment seriously.

As we can see in Figure 5, the curves are the speed tendency of optical encoder (a) and output current (b), in the case of open-loop vector control system with $600 \mathrm{rpm}$ rated speed and rotational speed is set to $17.35 \%$ of rated speed. Obviously, the speed curve approximates to the sine curve and its fluctuation amplitude reaches to $4.8 \mathrm{rpm}$.

Figure 6 is a part of enlargement of figure 5, and it shows the speed curve of encoder output in one cycle. To eliminate eccentric disturbance, the iterative algorithm of the simplified model is embedded into the control system speed feedback channel. And the corrected encoder output is shown in figure 7 . The corrected error is less than $0.5 \mathrm{rpm}$. Actual results prove that the elimination approach has a good correction effect, and can meet the general requirements of speed measurement.



Figure 5. Curve diagram of the encoder speed and output current. 


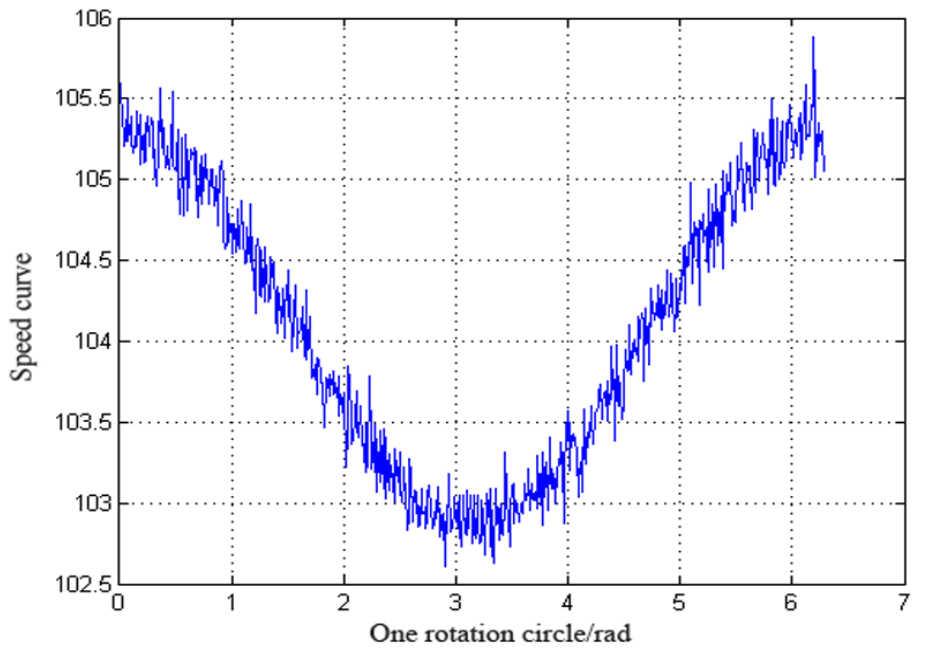

Figure 6. Curve of encoder speed.



Figure 7. Curve of encoder speed output after eccentricity correction.

\section{Conclusion}

By mean of analyzing the eccentric mechanism, this paper establishes the simplified model that can effectively eliminate the low-frequency disturbance caused by the eccentricity in the speed feedback channel. Finally, a high accuracy recursive algorithm which can be applied to digital control system is presented, and it has a higher application value. 


\section{Acknowledgments}

The authors wish to thank the financial support by the National Key R\&D Projects of China (2016YFF0102407).

\section{References}

1. X. Tang, W. Chen. A simple method about eliminating eccentricity error. Computation Technique. 7, 39-40(1996).

2. J. Tan, Y. Qin. Simulation Model of Optical Encoder and Estimation of Instantaneous Velocity for a Motor. Electric Drive. 2, 41-43(2001).

3. Y. Fang. Studies on digital addition technology in incremental encoder. Master's Thesis Graduate University of Chinese Academy of Sciences.2005.

4. R. Zhao, Z. Zhang, J. Tie. Influence of Encoder Eccentricity on Speed Measurement and Elimination Approach. International Conference on Network Computing and Information Security. 63-66 (2011).

5. X. Ma, R. Zhao. High precision rotating speed measuring device. China. Utility Models. ZL200720141299.8. 2008-3-12.

6. Z. Cai, R. Zhao, F. Cheng. Research on Digital Estimation Method of Motor Speed and Rotor Position. Electric Drive. 37(8), 12-14(2007).

7. Q. Yu, K. Liu, C. Wang, W. Yuan, W. Qian, C. Zhang. Choice of Optical-encoder and Measure of Speed and Rotor Place of Synchronous Motor. Electric Drive. 36(4), 17-20(2006).

8. D. Mancini, M. Brescia, E. Cascone, F. Cortecchia, P. Schipani. Encoder system design: strategies for error compensation. Proc. SPIE. 3351, 380-386(1998).

9. Y. Matsuzoe, N. Tsuji. Breadboard system and techniques to optimize the optical system of an encoder. Optical Engineering. 40(2), 283-288(2001). 